\title{
Degree of Contracture Related to Residual Muscle Shoulder Strength in Children with Obstetric Brachial Plexus Lesions
}

\author{
Valerie M. van Gelein Vitringa ${ }^{1} \quad$ Arthur van Noort $^{2}$ Marco J. P. F. Ritt ${ }^{3} \quad$ Barend J. van Royen ${ }^{1}$ \\ Johannes A. van der Sluijs ${ }^{1}$
}

${ }^{1}$ Department of Orthopaedic Surgery, Vu Medical Center, Amsterdam, The Netherlands

2 Department of Orthopaedic Surgery, Spaarne Ziekenhuis, Hoofddorp, The Netherlands

${ }^{3}$ Department of Plastic Surgery, VU Medical Center, Amsterdam, The Netherlands

J Brachial Plex Peripher Nerve Inj 2015;10:e23-e29.

\begin{abstract}
Address for correspondence Johannes A. van der Sluijs, MD, PhD, Department of Orthopaedic Surgery, VU Medical Center, Boelelaan 1117, PO Box 7057, 1007 MB Amsterdam, The Netherlands (e-mail: ja.vandersluijs@vumc.nl).
\end{abstract}

\begin{abstract}
Background and Objectives Little is known about the relation between residual muscle strength and joint contracture formation in neuromuscular disorders. This study aimed to investigate the relation between residual muscle strength and shoulder joint contractures in children with sequelae of obstetric brachial plexus lesion (OBPL). In OBPL a shoulder joint contracture is a frequent finding. We hypothesize that residual internal and external rotator strength and their balance are related to the extent of shoulder joint contracture.

Methods Clinical assessment was performed in 34 children (mean 10.0 years) with unilateral OBPL and Narakas classes I-III. External and internal rotation strengths were measured with the shoulder in neutral position using a handheld dynamometer. Strength on the affected side was given as percentage of the normal side. Contracture was assessed by passive internal and external rotations in degrees (in $0^{\circ}$ abduction). Mallet classification was used for active shoulder function.

Results External and internal rotation strengths on the affected side were approximately $50 \%$ of the normal side and on average both equally affected: $56 \%$ (SD 18\%)

\section{Keywords}

- obstetric brachial plexus lesion

- contracture

- muscle force/strength

- shoulder function respectively $51 \%($ SD $27 \%) ; r=0.600, p=0.000$. Residual strengths were not related to passive internal or external rotation $(p>0.200)$. Internal rotation strength $(r=$ $-0.425, p<0.05)$ was related to Narakas class. Mallet score was related to external and internal rotation strengths $(r=0.451$ and $r=0.515$, respectively; $p<0.01$ ). Conclusion The intuitive notion that imbalances in residual muscle strength influence contracture formation cannot be confirmed in this study. Our results are of interest for the understanding of contracture formation in OBPL.
\end{abstract}

received

August 5, 2014

accepted

June 3, 2015

published online

August 5, 2015
DOI http://dx.doi.org/

10.1055/s-0035-1558425.

ISSN $1749-7221$.
Copyright $\odot 2015$ Georg Thieme Verlag

KG Stuttgart - New York
License terms

()(1) $\Theta \circledast$ 


\section{Introduction}

An obstetric brachial plexus lesion (OBPL) is a lower motor neuron disorder, resulting from traction to the brachial plexus during labor and resulting in a palsy of the upper limb in varying extent. The incidence ranges from 0.42 to 5.1 in 1,000 live births. ${ }^{1,2}$ Although the majority of these children recover, in some children sequelae (paresis, joint contractures, and deformities) persist. ${ }^{3-5}$ Contractures of the shoulder, especially internal rotation contractures, are common in OBPL and are related to functional disability. ${ }^{2,6}$

The pathophysiology of contracture formation in OBPL is incompletely understood. Contracture formation starts with muscle abnormalities. According to an experimental mice study, ${ }^{7}$ denervation after neurectomy leads to reduced length and cross-sectional growth of the muscles. These growth differences were related to the contracture formation. Beside these growth differences qualitative changes as increased fibroses were found, although the relation between fibrosis and contractures was not strong.

Some of these muscle changes have also been found inconsistently in children with shoulder contractures after OBPL; Einarsson et al found reduced sarcomere length and increased fibrosis in the subscapular muscle after $\mathrm{OBPL},{ }^{8}$ but a later study found no abnormalities. ${ }^{6}$

In infants shoulder muscle atrophy was related to shoulder joint subluxation, ${ }^{9}$ but upper arm muscle atrophy was not related to residual muscle function. ${ }^{10}$

Data on the residual strength of affected muscles in neuromuscular disorders and their relation to joint contractures are scarce. A recent study found both reduced passive range of motion and muscle strength for internal and external rotations of the shoulder in children with OBPL, but the relation between range of motion and strength was not tested $^{11}$.

Muscle imbalance is a factor often assumed to be of importance in contracture formation in OBPL. ${ }^{3,4}$ Intuitively the notion that imbalance leads to contracture formation is attractive, but data to support it are scarce. In an experimental mice study, ${ }^{7}$ excision of the external rotators was not able to cause contractures, but in another mice study using temporary paresis ${ }^{12}$ with botulinum contractures occurred.

Little is known about residual strength of affected muscles in established joint contractures and whether there is a relation between the degree of contracture and muscle strength. In the Dupuytren hand flexion contracture of the fingers has been related to grip strength, ${ }^{13}$ but findings of this local soft tissue disorder cannot be extrapolated to contractures of the larger muscles in OBPL. In patients with stroke, strength and contracture of the elbow flexors were weakly related at 26 and 39 weeks but not after 1 year. ${ }^{14}$ Applying these findings to OBPL is debatable, because stroke is an upper motor neuron lesion generally occurring to full-grown adults. In OBPL the lower motor neuron lesion occurs at birth and contractures develop over time but do not affect all children with OBPL. ${ }^{2,3,6}$

According to the craniocaudal direction of the injury to the cervical nerve roots, one would expect the external rotators (C4-6) to be weaker than the internal rotators (C5-8), a situation assumed to lead to the often-described internal rotation contracture. ${ }^{3,4}$ Our hypothesis is that in OBPL internal rotators are stronger than external rotators and that the degree of joint contracture would be related to the balance between the residual strength of internal and external rotators.

These hypotheses were tested in a clinical study in children with OBPL, assessing the relation between passive shoulder rotations, residual muscle strength of internal and external rotators, and residual active shoulder function. ${ }^{15}$

\section{Methods}

\section{Participants}

Included were 34 consecutive children (20 boys and 14 girls) with unilateral OBPL with a mean age of 10.0 years, attending the regular, multidisciplinary checkup in the VUMC between December 1, 2009, and August 1, 2011. The extent of OPBL was classified according to Narakas. ${ }^{16}$ Excluded were children with Narakas class IV (total paresis of the arm) and patients younger than 4 years, because their cooperation on examination was expected to be poor. The study was in accordance with the rules of the medical ethical commission of the VUMC.

\section{Muscle Strength}

Isometric peak muscle strength was measured in Newton with the Microfet 2 dynamometer (Biometrics, Almere, The Netherlands) by the same orthopaedic surgeon on both normal and affected sides. Each measurement was repeated three times and the median value was noted. External and internal rotation strengths were measured standing upright with the shoulder in $0^{\circ}$ abduction and rotation as neutral as possible. The elbow was flexed to $90^{\circ}$, the lower arm in neutral degrees of pronation/supination. For external rotation the Microfet 2 was placed on the dorsal side of the distal forearm just proximal of the wrist. For internal rotation it was placed on the palmar side. The patients were requested to keep their upper body still while giving maximal pressure to the dynamometer ( $\mathbf{- F i g . 1}$ ). This method for isometric peak strength measurement has excellent validity. ${ }^{17}$ Strength on the affected side was given as percentage of the normal side to correct for age and interindividual differences.

To quantify the balance between residual internal and external rotator strengths, the ratio between the residual internal and external strengths was calculated and related to contracture formation.

\section{Contracture}

As a measure of shoulder joint contracture, passive rotations on the affected side were measured with a standard goniometer in standing position with the shoulder in $0^{\circ}$ abduction. For the internal rotation contracture, passive external rotation was measured. For external rotation contracture, passive internal rotation was measured.

\section{Clinical Outcome}

Active shoulder function was measured using the method of Mallet $^{15}$ (-Table 1). This score consists of five dimensions: 

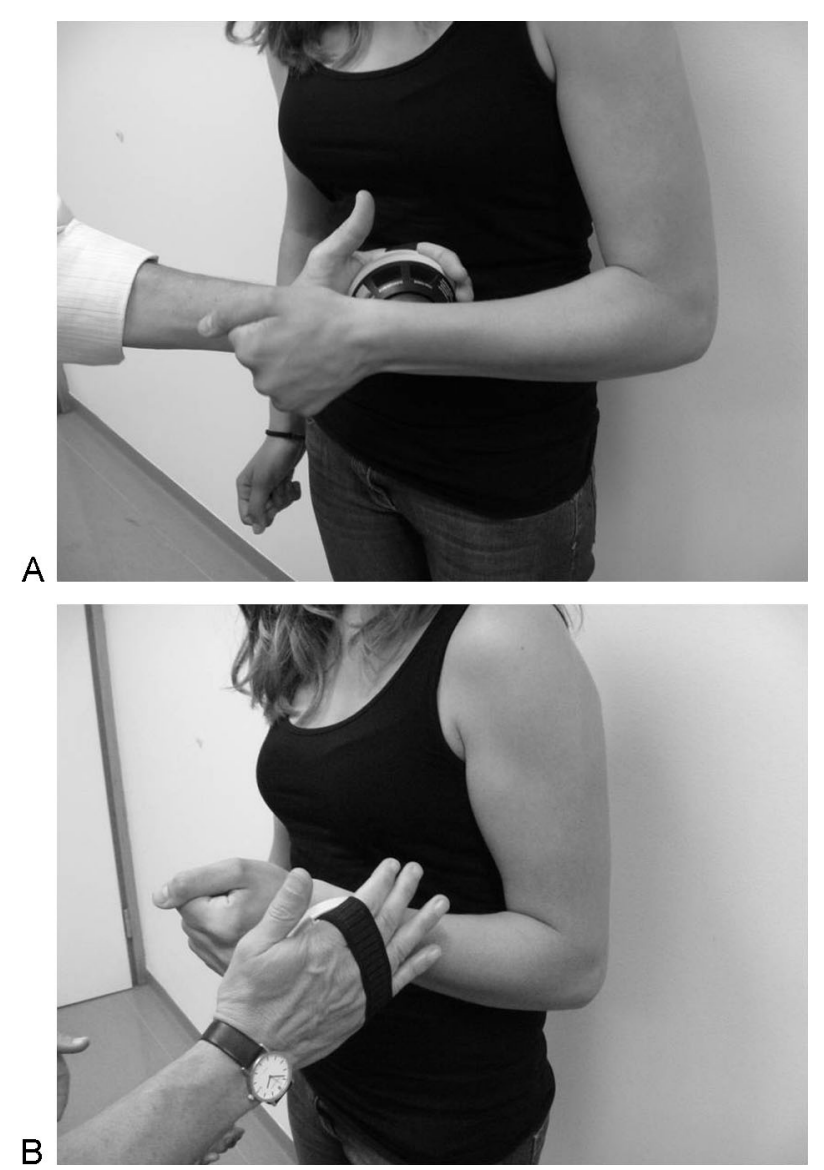

Fig. 1 Measurement of muscle strength. (A) Internal rotation strength is measured with the Microfet 2. (B) External rotation strength is measured.

abduction, external rotation, hand to neck, hand to back, and hand to mouth. We used the total Mallet score and the distinctive scores for external rotation and hand to back (the latter being a dimension for active internal rotation).

\section{Statistics}

All data were collected and analyzed in SPSS for Windows (version 15.0). Normal distribution was tested with Kurtosis and Skewness tests. Results are given as mean (standard deviation). Correlations were calculated using Pearson's for interval data or Spearman's for ordinal data test. Differences were tested using paired $t$-tests. All tests were two tailed and $p<0.05$ was considered to be significant.

\section{Results}

In this study 34 children with unilateral OBPL were included, with a mean age of 10.0 years (range $4.7-16.0$ years, standard deviation [SD] 1.5), 20 boys and 14 girls. The right side was affected in 21 children, the left in 13 . Narakas class was I in 19 children, II in 4 children, and III in 11 children, with a mean Narakas class of 1.8 (SD 1.0). In addition, 19 children had prior neurosurgery, 15 did not; 5 children have had secondary surgery around the shoulder. All data were normally distributed. Main descriptives and correlations are shown in $\mathbf{- T a b l e s} \mathbf{2}$ and $\mathbf{3}$, respectively.

\section{Muscle Strength}

Absolute muscle strength on the affected side was always less than that on the normal side, except for the internal rotation strength in one 11.8-year-old boy (106 and 102 Newton, respectively). There was no significant difference (paired $t$ test $p=0.26$ ) between mean residual external (56\%) and internal rotation strength $(51 \%)$ on the affected side (-Table 2). By approximation external and internal rotation strengths were both equally affected as shown in (- Fig. 2) and their correlation was significant ( - Table 3 ).

There was no relation between residual muscle strength and shoulder joint contracture ( - Table 3 ), or between the ratio of residual internal and external strength and joint contractures.

Muscle strength was related to the Mallet score ( - Table3). The Mallet subscore global external rotation was not related to muscle strength, whereas subscore hand to back was related to both muscle strengths (internal rotation $r=0.52$, external rotation $r=0.50 ; p<0.01$ ).

\section{Shoulder Joint Contracture}

Passive external and passive internal rotations were not interrelated ( - Table3). Passive external rotation was related to the total Mallet score, whereas passive internal rotation was not. Passive external rotation was related to the Mallet subscore global external rotation $(r=0.78, p=0.000)$, whereas passive internal rotation was related to subscore hand to back $(r=0.35, p=0.04)$.

Table 1 Measurement of active shoulder function according to Mallet

\begin{tabular}{|c|c|c|c|c|c|}
\hline Functional parameter & Class 1 & Class 2 & Class 3 & Class 4 & Class 5 \\
\hline Abduction & None & $<30^{\circ}$ & $30-90^{\circ}$ & $>90^{\circ}$ & Normal \\
\hline External rotation & None & $<0^{\circ}$ & $0-20^{\circ}$ & $>20^{\circ}$ & Normal \\
\hline Hand to neck & None & Not possible & Difficult & Easy & Normal \\
\hline Hand to back & None & Not possible & $\mathrm{S} 1$ & Th12 & Normal \\
\hline Hand to mouth & None & Marked trumpet sign ${ }^{a}$ & Partial trumpet sign ${ }^{a}$ & $<40$-degree abduction & Normal \\
\hline
\end{tabular}

Notes: Normal function in every dimension is 5 points, absent function is 1 point. Total Mallet score is the sum of the five sub-scores.

${ }^{a}$ Trumpet sing is abduction of the shoulder with simultaneous flexion of the elbow. 
Table 2 Descriptive of output values in mean, SD, range, and 95\% Cl

\begin{tabular}{|c|c|c|c|c|c|}
\hline & Descriptives & Mean & SD & Range & $95 \% \mathrm{Cl}$ \\
\hline & Age (month) & 120 & 32 & $57-191$ & $108-132$ \\
\hline & Narakas class & 1.8 & 1.0 & $1-3$ & $1.4-2.1$ \\
\hline \multirow[t]{2}{*}{ Normal side } & Internal rotation strength & $84 \mathrm{~N}$ & $26 \mathrm{~N}$ & $35-145 \mathrm{~N}$ & $75-94 N$ \\
\hline & External rotation strength & $64 \mathrm{~N}$ & $21 \mathrm{~N}$ & $27-130 \mathrm{~N}$ & $56-72 \mathrm{~N}$ \\
\hline \multirow[t]{2}{*}{ Affected side } & Internal rotation strength & $42 \mathrm{~N}$ & $26 \mathrm{~N}$ & $1-106 \mathrm{~N}$ & $32-51 \mathrm{~N}$ \\
\hline & External rotation strength & $35 \mathrm{~N}$ & $18 \mathrm{~N}$ & $15-76 \mathrm{~N}$ & $29-42 \mathrm{~N}$ \\
\hline \multirow[t]{2}{*}{ Ratio affected/normal } & Internal rotation strength & $51 \%$ & $27 \%$ & $0.01-1.04 \%$ & $41-60 \%$ \\
\hline & External rotation strength & $56 \%$ & $18 \%$ & $20-86 \%$ & $49-62 \%$ \\
\hline \multirow[t]{2}{*}{ Contracture } & Passive external rotation & $27^{\circ}$ & $34^{\circ}$ & $-40-90^{\circ}$ & $15-39^{\circ}$ \\
\hline & Passive internal rotation & $67^{\circ}$ & $18^{\circ}$ & $30-90^{\circ}$ & $61-74^{\circ}$ \\
\hline \multirow[t]{3}{*}{ Mallet score } & Total & 14.8 & 2.5 & $11-19$ & $14-16$ \\
\hline & External rotation & 2.6 & 0.8 & $2-4$ & $2.3-2.9$ \\
\hline & Hand to back & 3.0 & 0.9 & $2-4$ & $2.7-3.3$ \\
\hline
\end{tabular}

Abbreviations: $\mathrm{Cl}$, confidence interval; N, Newton; SD, standard deviation.

Table 3 Correlations between residual strength (\% of normal) and contractures. Shown are $r$ values, with p value subscribed

\begin{tabular}{|l|l|l|l|l|}
\hline & $\begin{array}{l}\text { External rotation } \\
\text { strength }\end{array}$ & $\begin{array}{l}\text { Internal rotation } \\
\text { strength }\end{array}$ & $\begin{array}{l}\text { Passive external } \\
\text { rotation }\end{array}$ & Passive internal rotation \\
\hline Internal rotation strength & 0.60 & & & \\
$\mathrm{P}=0.000$ & 0.22 & 0.07 & & \\
\hline Passive external rotation & 0.21 & 0.71 & & \\
\hline Passive internal rotation & 0.21 & 0.02 & -0.23 & 0.18 \\
\hline Total Mallet & 0.24 & 0.91 & 0.52 & 0.24 \\
& 0.45 & 0.42 & $\mathrm{P}=0.002$ & 0.17 \\
\hline
\end{tabular}

\section{Narakas Class and Age}

Age was related to all absolute muscle strengths ( $p<0.001)$, not to any of the other parameters. Narakas class was only related to internal rotation strength, not to any of the other parameters. In the higher and more severe Narakas classes, this internal rotation strength was weaker $(r=-0.43$, $p=0.01$ ). In the subgroup of children with Narakas class 1 , no relation was found between muscle strength and contracture ( - Table 4 ), neither a relation was found in the other classes.

\section{Discussion}

Our hypothesis that in OBPL internal rotators in the shoulder are stronger than external rotators and the hypothesis that the balance between residual muscle strength of internal and external rotators is related to the internal rotation contracture were not confirmed.

There are several limitations of our study. The first limitation is that it is not clear which muscles were measured. Several muscles contribute to rotation of the shoulder, but their individual contribution is not fully known. Based on electromyographic (EMG) studies in healthy patients, subscapularis and, to a lesser extent, pectoralis major are predominantly active during internal rotation while supra- and infraspinatus, trapezius, and serratus muscles are active during external rotation. ${ }^{18,19}$ It is unknown whether the relative contributions of the muscles in the OBPL child are the same. Changes in lateral myofascial strength transmission might possibly lead to changes in the relative contribution of the various muscles to rotation strengths. ${ }^{20,21}$

Another limitation is the measurement of muscle strength. Measurement using a handheld dynamometer have been found to be reliable. ${ }^{17}$ In our study we used the median of three consecutive isometric measurements. However, isometric peak strength is among others dependent on muscle length. ${ }^{21}$ Though this was standardized by keeping the arm in neutral external rotation if possible, differences in muscle resting length in this position between the children might have influenced the results. Given the presence of contractures, the neutral position probably does not standardize the muscle length of the internal rotators in these children and in some they are stretched beyond their resting length, thus influencing peak muscle strength. The duration of the peak is 


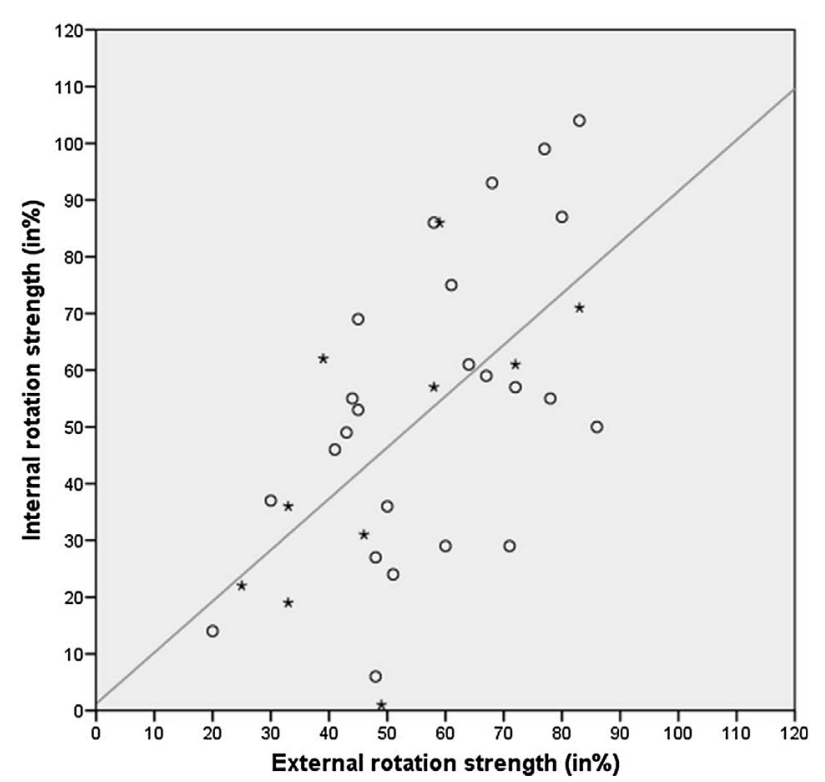

Fig. 2 Significant relation between external and internal rotation strengths $(r=0.60, p=0.00)$. The distribution of children with severe internal rotation contractures $\left({ }^{*}\right.$, passive external rotation $\leq 0^{\circ}$ ) and children with mild contractures $\left(\mathrm{O}\right.$, passive external rotation $\left.>0^{\circ}\right)$ is shown. Children with severe contractures are evenly distributed, and there is no relation between contracture and strength.

neither considered by the handheld dynamometer with differences in endurance not being measured. Peak values might be possibly subservient contributors to the function of these partially denervated muscles.

Another limitation is that the original lesion that predominantly affects the upper trunk (the external rotators) and that was the basis of our hypothesis on residual strength was influenced by the neurosurgery that was performed in the majority of patients (19 of 34). Finally, the contralateral side may have hypertrophied to compensate for the paretic arm, thus increasing the difference between affected and unaffected.

In spite of these caveats, peak strength measurement seems to measure a valid aspect of OBPL as it was related with Narakas class, a measure of the neurologic trauma, and with clinical outcome of OBPL as assessed by total Mallet score. We found that the Narakas class correlated with internal rotation strength but not with external rotator strength. Children with higher Narakas classes had weaker internal rotation strength than those with lower classes. This makes sense as in Narakas class I only C5 and 6 are affected, and the subscapular muscle (internal rotator) is innervated by the subscapular nerves from C5 to 8. In higher Narakas classes in which $\mathrm{C7}$ and $\mathrm{C} 8$ too are affected, internal rotation strength is weaker. As the external rotators (mostly $\mathrm{C} 5$ and 6 ) are affected in all Narakas classes, this explains why there was no relation between Narakas class and external rotation strength.

Based on the craniocaudal direction of the injury to the cervical nerve roots, we expected the external rotation strength (C4-6) to be weaker than internal rotation (C5-8). However, we found on average no difference between residual internal rotation strength (51\% of normal side) and residual external rotation strength (56\% of normal). In a substantial number of patients internal rotators were weaker than external rotators. Our results are not confirmed by the scarce literature; the Kirjavainen et al's study ${ }^{11}$ found less residual strength in external rotator ( $28 \%$ of the contralateral side) than in internal rotators ( $44 \%$ of the contralateral side). Possibly the fact that $50 \%$ of their population had had secondary shoulder surgery (either soft tissues or humeral osteotomy) may have influenced their results although this would lead to a decrease in internal rotator strength, which is not what they found. Their measurement technique was different, but their shoulder position was identical. Part of the different results could be explained because they used the best of three values, in our study the median.

The lack of difference between internal and external rotators despite the fact that the neurologic lesion to the external rotators is larger is intriguing. Possibly the muscles were not measured on identical lengths in their strength length curves. In children with an internal rotation contracture, the neutral position lengthens the internal rotators and shortens the external rotators. The measurement points on the strength length curves would differ: internal is shifted to the right, external to the left. This bias would, however, lead to higher values for the internal rotators, which is not what we found.

The strength length curves of the affected muscles are probably different and the weakest muscles may reorganize their architecture to optimize their peak strength. The internal rotation contracture itself might be a symptom of this process. It stretches the external rotators and shortens the internal rotators, thus shifting their strength length curves in a way that increases external rotation strength and reduces

Table 4 Correlations in subgroup of children with Narakas class $1(n=19)$

\begin{tabular}{|l|l|l|l|}
\hline & External rotation strength & Internal rotation strength & Passive external rotation \\
\hline Internal rotation strength & $\mathbf{0 . 6 7}$ & & \\
& $\mathbf{0 . 0 0 2}$ & & \\
\hline Passive external rotation & 0.27 & -0.003 & \\
& 0.27 & 0.99 & -0.34 \\
\hline Passive internal rotation & 0.26 & 0.28 & 0.16 \\
\hline
\end{tabular}

Note: Correlations are shown in $r$ value, with $p$ value subscribed. 
internal rotation strength. In this context the usual adaptation of the number of sarcomeres after shortening ${ }^{22,23}$ possibly does not seem to occur as muscle strength of the internal rotators is permanently reduced compared with normal.

Possibly increased fiber pennation, more parallel fibers, increased lateral strength transmission, from other muscles, and reduced tendon elasticity all contribute to maximization of peak strength in the external rotators. ${ }^{21}$ Alternatively, the internal rotators might possibly adapt to a reduced function of their antagonist by reduced growth because of inadequate stretching by the antagonist. This reduced growth might reduce their function. Both mechanisms might be responsible for the described balancing of the residual strengths around the shoulder.

As far the role of strength imbalance and contractures: in shoulders of children with OBPL, muscle imbalance is often mentioned as (or thought to be) a cause of secondary shoulder deformities. ${ }^{24-26}$ Muscle imbalance after experimental neurectomy in mice causes contractures. ${ }^{7}$ Hogendoorn et $\mathrm{al}^{24}$ suggest that muscle imbalance in less affected OBPL children contributes to shoulder deformity and that in more severe OBPL cases the absent imbalance protects these shoulders from severe deformity. However, we did not find a muscle imbalance in our study; even in our subgroup of children with Narakas class I, internal and external rotation strengths were equally affected and related.

The relation between strength imbalance and contractures is weak. Although on average there is no imbalance in peak muscle strength between internal and external rotations, in most children an imbalance is present, as shown in (-Fig. 2), but this imbalance could not predict extent of contracture. The role of strength in contracture formation is further questioned by an experimental study ${ }^{7}$ where strength imbalance created by resection of the external rotator was not a dominant factor in contracture formation.

Based on experimental and clinical studies, differences in muscle growth seem important in the causation of contractures. In children with OBPL muscle volume imbalance has been described with the subscapularis muscle (internal rotator) being more affected than the infraspinatus (external rotator). 5,9,25,27 In the cited neurectomy study ${ }^{7}$ subscapularis and elbow flexors had reduced longitudinal growth that correlated with contracture formation. Qualitative muscle changes also play a role. In the cited experimental study, muscle fibrosis was present, but the correlation with contractures not consistent. In OBPL children Einarsson et al found reduced sarcomere length and increased fibrosis in the subscapular muscle ${ }^{8}$ although, given the nature of this controls (other upper arm muscles), the changes in sarcomere length have to be interpreted with care and a later study from the same group found that muscle biopsies overall showed normal morphology. ${ }^{6}$ Structural muscle size abnormalities on magnetic resonance imaging (MRI) level in subscapularis and infraspinatus correlated with glenoid deformity and humeral head subluxation. $^{24}$

Reduced length growth of partially denervated muscles is an important factor in the pathophysiology of contracture formation, while our results do not confirm that imbalances in residual muscle strength seem to be relevant.

\section{Conclusion}

In our study there was no relation found between shoulder joint contracture and residual muscle strength of internal and external rotators in OBPL. Muscle strengths of internal and external rotation were on average equally affected. The intuitive notion that imbalances in residual muscle strength lead to an internal rotation contracture of the shoulder cannot be confirmed in this study. Reductions in growth rate in partially denervated muscles are more likely causes of contractures.

\section{Funding}

There was no financial support received.

\section{References}

1 Evans-Jones G, Kay SP, Weindling AM, et al. Congenital brachial palsy: incidence, causes, and outcome in the United Kingdom and Republic of Ireland. Arch Dis Child Fetal Neonatal Ed 2003;88(3): F185-F189

2 Hoeksma AF, Ter Steeg AM, Dijkstra P, Nelissen RG, Beelen A, de Jong BA. Shoulder contracture and osseous deformity in obstetrical brachial plexus injuries. J Bone Joint Surg Am 2003;85-A(2): 316-322

3 Hale HB, Bae DS, Waters PM. Current concepts in the management of brachial plexus birth palsy. J Hand Surg Am 2010;35(2):322-331

4 Pearl ML. Shoulder problems in children with brachial plexus birth palsy: evaluation and management. J Am Acad Orthop Surg 2009; 17(4):242-254

5 Waters PM, Monica JT, Earp BE, Zurakowski D, Bae DS. Correlation of radiographic muscle cross-sectional area with glenohumeral deformity in children with brachial plexus birth palsy. J Bone Joint Surg Am 2009;91(10):2367-2375

6 Hultgren T, Einarsson F, Runesson E, Hemlin C, Fridén J, Ljung BO. Structural characteristics of the subscapularis muscle in children with medial rotation contracture of the shoulder after obstetric brachial plexus injury. J Hand Surg Eur Vol 2010;35(1):23-28

7 Nikolaou S, Peterson E, Kim A, Wylie C, Cornwall R. Impaired growth of denervated muscle contributes to contracture formation following neonatal brachial plexus injury. J Bone Joint Surg Am 2011;93(5):461-470

8 Einarsson F, Hultgren T, Ljung BO, Runesson E, Fridén J. Subscapularis muscle mechanics in children with obstetric brachial plexus palsy. J Hand Surg Eur Vol 2008;33(4):507-512

9 Van Gelein Vitringa VM, Jaspers R, Mullender M, Ouwerkerk WJ, Van Der Sluijs JA. Early effects of muscle atrophy on shoulder joint development in infants with unilateral birth brachial plexus injury. Dev Med Child Neurol 2011;53(2):173-178

10 Ruoff JM, van der Sluijs JA, van Ouwerkerk WJ, Jaspers RT. Musculoskeletal growth in the upper arm in infants after obstetric brachial plexus lesions and its relation with residual muscle function. Dev Med Child Neurol 2012;54(11):1050-1056

11 Kirjavainen MO, Nietosvaara Y, Rautakorpi SM, et al. Range of motion and strength after surgery for brachial plexus birth palsy. Acta Orthop 2011;82(1):69-75

12 Kim HM, Galatz LM, Patel N, Das R, Thomopoulos S. Recovery potential after postnatal shoulder paralysis. An animal model of neonatal brachial plexus palsy. J Bone Joint Surg Am 2009;91(4): 879-891 
13 Zyluk A, Jagielski W. The effect of the severity of the Dupuytren's contracture on the function of the hand before and after surgery. J Hand Surg Eur Vol 2007;32(3):326-329

14 Ada L, O'Dwyer N, O'Neill E. Relation between spasticity, weakness and contracture of the elbow flexors and upper limb activity after stroke: an observational study. Disabil Rehabil 2006;28(13-14): 891-897

15 Mallet J. Obstetrical paralysis of the brachial plexus. II. Therapeutics. Treatment of sequelae. Priority for the treatment of the shoulder. Method for the expression of results [in French]. Rev Chir Orthop Repar Appar Mot 1972;58:1, 166-168

16 Narakas AO. Obstetrical brachial plexus injuries. In: Lamb D, ed. The Paralysed Hand. Edinburgh, UK: Churchill Livingstone; 1987:116-135

17 Hébert LJ, Maltais DB, Lepage C, Saulnier J, Crête M, Perron M. Isometric muscle strength in youth assessed by hand-held dynamometry: a feasibility, reliability, and validity study. Pediatr Phys Ther 2011;23(3):289-299

18 Boettcher CE, Cathers I, Ginn KA. The role of shoulder muscles is task specific. J Sci Med Sport 2010;13(6):651-656

19 Escamilla RF, Yamashiro K, Paulos L, Andrews JR. Shoulder muscle activity and function in common shoulder rehabilitation exercises. Sports Med 2009;39(8):663-685

20 Huijing PA, Jaspers RT. Adaptation of muscle size and myofascial force transmission: a review and some new experimental results. Scand J Med Sci Sports 2005;15(6):349-380
21 Zatsiorsky VM, Prilutsky BI. Biomechanics of Skeletal Muscles. Leeds, UK: Human Kinetics; 2012

22 Williams PE, Goldspink G. Changes in sarcomere length and physiological properties in immobilized muscle. J Anat 1978; 127(Pt 3):459-468

23 Koh TJ, Herzog W. Excursion is important in regulating sarcomere number in the growing rabbit tibialis anterior. J Physiol 1998;508 (Pt 1):267-280

24 Hogendoorn S, van Overvest KL, Watt I, Duijsens AH, Nelissen RG. Structural changes in muscle and glenohumeral joint deformity in neonatal brachial plexus palsy. J Bone Joint Surg Am 2010;92(4): 935-942

25 Pöyhiä TH, Nietosvaara YA, Remes VM, Kirjavainen MO, Peltonen JI, Lamminen AE. MRI of rotator cuff muscle atrophy in relation to glenohumeral joint incongruence in brachial plexus birth injury. Pediatr Radiol 2005;35(4):402-409

26 Waters PM, Smith GR, Jaramillo D. Glenohumeral deformity secondary to brachial plexus birth palsy. J Bone Joint Surg Am 1998;80(5):668-677

27 van Gelein Vitringa VM, van Kooten EO, Jaspers RT, Mullender MG, van Doorn-Loogman MH, van der Sluijs JA. An MRI study on the relations between muscle atrophy, shoulder function and glenohumeral deformity in shoulders of children with obstetric brachial plexus injury. J Brachial Plex Peripher Nerve Inj 2009; $4: 9$ 